\title{
Rootstock Effects on Pistachio Trees Grown in Verticillium dahliae-Infested Soil
}

\author{
L. Epstein, R. Beede, S. Kaur, and L. Ferguson
}

First and third authors: Department of Plant Pathology, University of California, Davis 95616; second author: University of California Cooperative Extension Kings County, 680 N Campus Dr., Suite A, Hanford, CA 93230; and fourth author: Department of Pomology, University of California, Davis 95656.

Accepted for publication 29 November 2003.

\begin{abstract}
Epstein, L., Beede, R., Kaur, S., and Ferguson, L. 2004. Rootstock effects on pistachio trees grown in Verticillium dahliae-infested soil. Phytopathology 94:388-395.

In a field trial in soil infested with Verticillium dahliae, we compared the yield, growth, incidence of symptoms of Verticillium wilt, and mortality of two interspecific hybrid pistachio tree rootstocks (UCBI and PGII) with the standard rootstocks: the $V$. dahliae-resistant and susceptible Pistacia integerrima and $P$. atlantica, respectively. After 10 years, the trees were destructively sampled for $V$. dahliae in the xylem at the graft union. The results indicate that trees on the $(P$. atlantica 'KAC' $\times P$. integerrima) hybrid UCBI rootstock grew and yielded as well as those on
\end{abstract}

ABSTRACT

Commercial pistachio nut production started in California in 1976. In 2003, there were approximately 34,000 bearing ha primarily in the central and southern portion of the San Joaquin Valley. The plantings initially used Pistacia atlantica rootstocks (21). However, P. atlantica is susceptible to infection by Verticillium dahliae, the causal agent of Verticillium wilt. V. dahliae is endemic in San Joaquin Valley soils and the fungus has an extremely wide host range (22). Moreover, cotton is a prodigious producer of microsclerotia of $V$. dahliae and many of the pistachio trees were planted in soil previously used to grow cotton (4). While infection occurred on pistachio trees planted in virgin sites, tree loss on former cotton ground was economically devastating. Consequently, Verticillium wilt rapidly became the most important disease of pistachios (4).

Verticillium wilt can be mitigated by reducing microsclerotial inoculum either by preplant fumigation (4) or by preplant or inseason solarization (3). Following the identification of resistance to Verticillium wilt in pistachios (23) starting in 1982, new pistachio plantings in regions with a history of Verticillium wilt were generally planted on $P$. integerrima 'Pioneer Gold' rootstocks (21). Although $P$. integerrima is comparatively unaffected by Verticillium wilt, the rootstock is sensitive to frost. In the mid1980s, two interspecific hybrid pistachio rootstocks were introduced into California: 'Pioneer Gold II' (PGII) $(P$. atlantica $\times P$. integerrima) and 'UCBI' ( $P$. atlantica 'KAC' $\times P$. integerrima $)$. Based on a combination of results from a 1-year field trial in 1990 and laboratory assays, Morgan et al. (20) concluded that compared with $P$. integerrima and $P$. atlantica as resistant and susceptible standards, respectively, UCBI was moderately resistant to resistant and PGII was susceptible to Verticillium wilt. In

Corresponding author: L. Epstein; E-mail address: lepstein@ucdavis.edu

Publication no. P-2004-0130-02R

(C) 2004 The American Phytopathological Society
P. integerrima. Trees on the hybrid PGII yielded the least. Analysis of variance and log-linear models indicate that in soil infested with $V$. dahliae, three associations significantly affect pistachio nut yield. Rootstock affects scion vigor and extent of infection. Third, the extent of infection and scion vigor are inversely associated. Although trees on the $P$. integerrima rootstock had the highest ratings in a visual assessment of vigor, $65 \%$ were infected with $V$. dahliae in the trunk in the graft region compared with $73 \%$ in $P$. atlantica and $25 \%$ in UCBI. Thus, $P$. integerrima and UCBI have at least one different mechanism for resistance to $V$. dahliae.

Additional keyword: nut trees.

1992, we started a 10-year field trial in soil naturally infested with $V$. dahliae. The purpose of this research was to compare the incidence of infection and the horticultural performance of pistachio nut trees planted on UCBI rootstocks with those planted on P. integerrima, $P$. atlantica, and PGII.

\section{MATERIALS AND METHODS}

Plant material. Pistachios are dioecious. P. integerrima 'Pioneer Gold', P. atlantica, and 'Pioneer Gold II' seedlings were obtained commercially; all are produced by open-pollination. 'UCBI' seeds (Foundation Plant Materials Service, University of California, Davis) are produced so that there is a specific pollen parent. For the 10-year trial, 1-year-old seedlings of the four rootstocks were budded in the nursery. The rootstock seedlings used for nut production were grafted with buds of the vegetatively propagated $P$. vera 'Kerman' tree. Similarly, the seedlings used as pollinator trees were grafted with buds from the vegetatively propagated $P$. vera 'Peters' tree. Thus, all genetic differences in nut production were due to differences in the rootstocks.

Field trial. The trial was conducted on a Panoche sandy clay loam at the Westside Research and Extension Center, University of California, Five Points. The field history before 1990 included cotton and tomato plant cultivation, with typical symptoms of Verticillium wilt. In January 1990, 1-year-old seedlings were transplanted into a planting with 18 rows and 18 trees per row. Trees were spaced $5 \mathrm{~m}$ apart in $6 \mathrm{~m}$ wide rows. The trees on the borders were not used in the study. In December 1990, a killing frost caused tree damage. Thus, the trees were removed in 1991, the soil was redistributed by plowing, and a second trial was planted in 1992. Trees were planted in a completely randomized block design with 64 blocks, with each block extending across four rows and having one tree of each of the four rootstocks in a random order. In 19 of the 256 positions for trees, trees with male scions were planted. Trees were maintained according to "best 
management practices" recommended by the University of California Cooperative Extension. In order to minimize the presence of alternate hosts for $V$. dahliae, weeds were routinely controlled with herbicides.

Assessment of response variables. Each year, we recorded trunk circumference at $46 \mathrm{~cm}$ above the soil, presence of symptoms characteristic of Verticillium wilt, and a categorical assessment of tree vigor as either dead, poor, fair, good, or excellent. Tree vigor was visually assessed in late September, after nuts were harvested so that vigor ratings were not confounded by yield. Vigor assessment was based on canopy size, density and color of foliage, and number and length of new shoots. Fungal isolations were made from symptomatic tissue when the sampling did not interfere with the experimental design, i.e., when a tree died or when a symptomatic branch would normally be pruned. Nut yield and quality were measured annually after bearing commenced in 1998. In 1998 and 1999, pistachio fruits were harvested and a 200-g aliquot from each tree was sorted into the following categories of nuts: undamaged splits, nonsplits, blanks, and damaged. After each component was weighed, marketable yield per tree was calculated as kilogram fresh weight harvested $x$ fraction of undamaged, in-shell split nuts $\times 0.35$; the 0.35 multiplier adjusts for an average loss of weight due to exocarp removal and drying nuts to 5\% moisture. From 2000 to 2002, fresh nuts from each tree were weighed separately as before, but assessments of marketable yield were made by a commercial processor (Paramount Growers, Lost Hills, CA). For this assessment, samples from trees on the same rootstock from eight blocks, i.e., with a maximum of eight trees for each rootstock per block, were pooled. For each of the eight replicates per rootstock, a 9-kg composite sample was hulled, dried to approximately $5 \%$ moisture, assessed for percent moisture, and weighed. Then, a 500-g sample was sorted and weighed as indicated previously. Marketable yield per tree was calculated as kilogram fresh weight harvested $\times$ fraction of weight in dry, undamaged, in-shell split nuts adjusted for $5 \%$ moisture.

Assessment of infection. After harvest in 2002, the plot was sampled destructively. A cross section of wood extending $5 \mathrm{~cm}$ above and below the graft union was removed from each tree. After the wood was polished, the images of the cut surfaces were recorded as digital files. Using SigmaScan Pro 5 Image Analysis software (SPSS, Chicago, IL), we traced the outline of the discolored portion of the wood and the perimeter of the wood. The area of the nondiscolored wood and the percentage of nondiscolored wood were calculated.

Each wood disk was examined macroscopically on the scion and rootstock for visual indications of infection by $V$. dahliae. Each surface was rated as having either no typical symptoms, characteristic symptoms in five or fewer spots on the wood, or symptoms in six or more spots. A minimum of five isolations were made from each side of the disk from every tree. Most isolations were from symptomatic regions. In the absence of symptoms, isolations were made from nonsymptomatic xylem. The wood was surface-sterilized with $0.5 \% \mathrm{NaOCl}$ in $0.01 \%$ Tween 20. After $2 \mathrm{~min}$, the surface layer of wood was removed with a blade and an internal piece was excised and plated on $1.5 \%$ water agar containing $0.01 \%$ chloramphenicol. After incubation at $24^{\circ} \mathrm{C}$ for 14 days, the plates were examined under a dissecting microscope for $V$. dahliae; the fungus was identified by its characteristic chains of microsclerotia.

Microsclerotial concentration in soil. The field was divided into four blocks each encompassing four rows of the trial. Within each row, three tree positions were selected with a random number generator and a soil core (48-mm diameter and $152 \mathrm{~mm}$ deep) was removed within the row, $1 \mathrm{~m}$ from the trunk of the designated tree. The 12 soil cores from each block were pooled into a single sample. Soil was air-dried to kill $V$. dahliae conidia and then ground to a particle size of $<2 \mathrm{~mm}$. Ten determinations of micro- sclerotial density were made per block. For each determination, $20 \mathrm{~g}$ of soil was suspended in $100 \mathrm{ml}$ of water (NANOpure II, Barnstead) and aliquots of $0.2 \mathrm{~g}$ of soil were distributed onto each of 10 pectate agar plates (15). The medium contained (per liter of $\left.10 \mathrm{mM} \mathrm{KHPO}{ }_{4}[\mathrm{pH} 7]\right) 4 \mathrm{~g}$ of sodium polypectate, $275 \mathrm{mg}$ of $\mathrm{MgSO}_{4} \cdot 7 \mathrm{H}_{2} \mathrm{O}, 1 \mathrm{~g}$ of $\mathrm{KNO}_{3}, 1 \mathrm{mg}$ of biotin, $1 \mathrm{ml}$ of Tergitol NP10 , and $15 \mathrm{~g}$ of agar. After adjusting $\mathrm{pH}$ to 7 and autoclaving, molten agar was amended for a final concentration of $0.25 \%$ chloramphenicol and $0.2 \%$ each chlortetracycline, penicillin $\mathrm{G}$, and streptomycin sulfate. After incubation at $21^{\circ} \mathrm{C}$ for 14 to 20 days, soil was washed off of the plates, and the agar was examined under a dissecting microscope for colonies with V. dahliae microsclerotia.

Statistical analyses. Data were analyzed using Data Analysis and Presentation ([DAP] GNU/Linux, Free Software Foundation, Boston, MA). Circumference and yield data were examined for nonnormality and heterogeneity of variance before analysis of variance (ANOVA) (16). For both response variables, only models with a significant $F$ value were analyzed further. All significant interactions are noted. When interaction terms were nonsignificant, they were removed from the model. Least-squares means were used in comparisons of treatments with different cell sizes. Mean comparisons of circumference and yield between rootstocks were made by Tukey's method $(P=0.05)$.

The effect of rootstock and one of three response variables (disease severity, vigor, and symptomatic trunks) on yield was analyzed with a two-factor ANOVA. The caveat is given that the categorical response variables were not the result of randomly assigned treatments. In addition, there is some measurement error with disease severity and vigor from categorization of a continuous variable. Levels of disease severity were analyzed with contrasts. When multiple contrasts were made, the acceptable $P$ value $(P=$ 0.05) was reduced using a Bonferroni-adjusted criterion (19). After vigor was assessed as described previously, effect of levels of vigor was analyzed by linear regression using the following assigned values: $2=$ poor, $3=$ fair, $4=$ good, and $5=$ excellent.

For analysis of vigor by ANOVA, the same values were assigned as indicated previously with $1=$ dead. For analysis of incidence data, the trees in eight contiguous blocks were pooled so that the data were analyzed for a design with 8 rather than 64 blocks. The fraction of trees in the designated category per block was transformed by arcsine $(\sqrt{ } \mathrm{x})$. However, the vigor and incidence data did not meet the assumptions for ANOVA because the errors were not normally distributed by the Shapiro-Wilks test. In addition, the transformed percentage data were heteroscedastic. Also, we note that the $P$ values computed from ANOVA are based on the assumption that the response variable is continuous. Consequently, vigor and incidence data also were analyzed by loglinear models (1), which are a method of categorical data analysis. For log-linear models, when indicated, categories were pooled to achieve a larger cell size. When simultaneous inferences were made for categorical analyses of data shown as percentages, the acceptable $P$ value $(P=0.05)$ was reduced using a Bonferroniadjusted criterion. Gabriel's (12) method for multiple comparisons gave the same results as Bonferroni.

\section{RESULTS}

When the soil was originally prepared for the trial in 1990, the field contained $40 \pm 1$ (standard error) microsclerotia per $\mathrm{g}$ of airdried soil. After 4 years without cotton or tomatoes in a relatively weed-free field, the microsclerotia concentration decreased 10 -fold to $4 \pm 0.4$ microsclerotia per $\mathrm{g}$. The microsclerotia concentration remained at less than 5 microsclerotia per $\mathrm{g}$ of soil through 1999. In 2002, the soil had $7 \pm 2$ microsclerotia per $g$ of soil.

Four pistachio rootstocks ( $P$. integerrima, $P$. atlantica, and two interspecific hybrids) were planted in a field in soil infested with V. dahliae in 1990 (20). In November 1990, all of the P. integer- 
rima and the UCBI were alive. In December, minimum temperatures for 11 nights ranged from -12 to $-4^{\circ} \mathrm{C}$. In late April 1991, $41 \%$ of the $P$. integerrima were dead in contrast to none of the UCBI $\left(\chi^{2}=32.6 ; P=0.0001\right)$. The surviving trees were assessed for injury; trees were rated as damaged if they had dead buds and a brown discoloration of the cambium at the soil line. Seventytwo percent of the surviving trees on $P$. integerrima rootstocks had symptoms consistent with frost injury in contrast to $4 \%$ of the UCBI $\left(\chi^{2}=50.6 ; P=0.0001\right)$. Thus, pistachio trees grown on hybrid UCBI are frost-tolerant, whereas those on $P$. integerrima are frost-sensitive.

In the 10-year trial, 18 female and no male trees died; all deaths were on either $P$. atlantica or PGII rootstocks (Table 1). The deaths occurred over the course of the study, with an annual loss of $1.7 \%$ for $P$. atlantica and $1.1 \%$ for PGII. Among the surviving trees, significantly more of the $P$. atlantica and PGII had the classic symptoms of Verticillium wilt compared with $P$. integerrima and UCBI. These symptoms include a "strike" in which leaves on one or more branches on one side of a tree wilt suddenly in late spring or summer and then die or "thin leaf decline" in which infected trees have off-colored leaves, poor terminal shoot growth, and thin canopies (9). The rankings of the rootstocks for vigor were fairly consistent over time, and the distribution of vigor shown for 2002 (Table 1) is representative of the other years (data not shown).

Due to the deaths in only two of the rootstocks, and a random and nonuniform number of males per rootstock, the number of surviving female trees per rootstock varied, with 51 PGII, 53 $P$. atlantica, 55 P. integerrima, and 61 UCBI. Data on circumfer- ence, vigor, and infection were analyzed for all trees with no evidence of a difference in response to $V$. dahliae between $P$. vera female 'Kerman' and male 'Peters' scions (data not shown). However, because marketable yield was our primary response variable, the data shown in Tables 2 to 6 are limited to female trees that survived through 2002.

All the scions (marked S in Fig. 1) were genetically identical. The center of all scions is gray to brown. In uninfected scions (Fig. 1A), only the first 5 or 6 years of wood is discolored and the margin of the region is even. Infected scions (Fig. 1B, C, D, G, and I) often have an irregular margin that frequently extends to a darker region from which $V$. dahliae was readily isolated. $V$. dahliae also was isolated from the irregular pigmented region, although less frequently than from the darker margin.

$V$. dahliae, on either the rootstock or the scion, was readily isolated from brown or black lesions in the xylem; representative lesions are shown with arrows in Figure 1. The lesions were in both spring and summerwood, but more frequently in springwood. While the characteristic brown or black lesions were the same on all rootstocks, the pigmentation pattern between rootstocks on both noninfected and infected rootstocks was more variable than on the scions. $P$. integerrima and PGII had a pigmentation pattern similar to the scion, although they lacked the uniform pigmentation in the center. The pigment produced by $P$. atlantica and UCBI tended to be blacker, more oblong, and independent of infection.

Isolations were made from both symptomatic and asymptomatic portions of both the scion and the rootstock side of the wood at the graft union. Of the 237 surviving trees, $74 \%$ of the

TABLE 1. Growth, yield, and incidence of symptoms of Verticillium wilt in pistachio trees planted on different rootstocks in soil naturally infested with Verticillium dahliae $^{\mathrm{s}}$

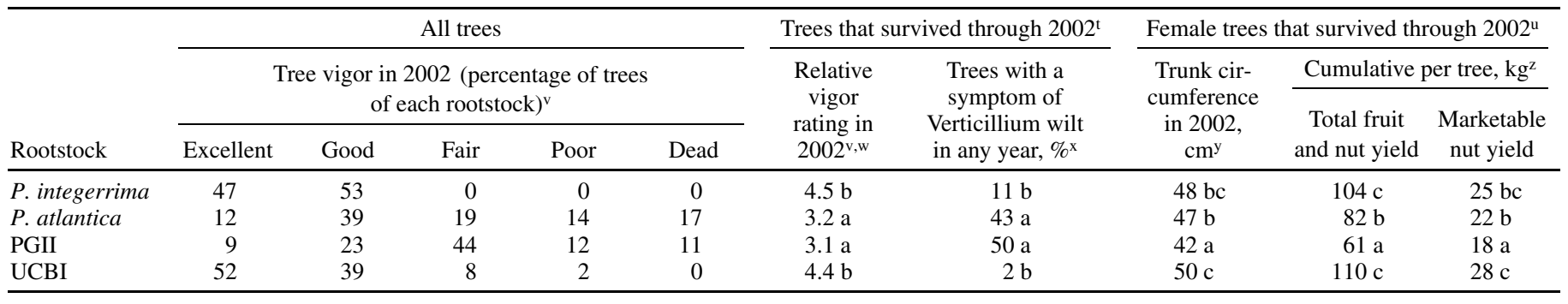

${ }^{\mathrm{s}}$ Sixty-four trees per rootstock were planted in 1992 in the San Joaquin Valley in California. Pistacia integerrima and P. atlantica are the V. dahliae-resistant and susceptible standards, respectively.

${ }^{t}$ Means followed by the same letter are not significantly different $(P \geq 0.05)$. Groupings were the same for both analysis of variance and log-linear models.

"All values are per female tree. Within a column, values followed by the same letter are not significantly different according to Tukey's method $(P=0.05)$.

${ }^{v}$ Vigor was rated using a visual assessment of canopy size, density and color of foliage, and number and length of new shoots. A log-linear model $(P=0.05)$ adjusted by a Bonferroni criterion indicated that $P$. integerrima $\equiv \mathrm{UCBI}>P$. atlantica $\equiv$ PGII. . dahliae was the apparent cause of all deaths.

${ }^{\mathrm{w}}$ Values are based on a number assignment of vigor ratings with $2=$ poor, $3=$ fair, $4=$ good, and $5=$ excellent.

x Trees with "strike" or "thin leaf decline" in at least 1 year were rated as symptomatic.

${ }^{y}$ Circumference was measured $46 \mathrm{~cm}$ above the soil.

${ }^{\mathrm{z}}$ Yields were collected from first bearing in 1998 through 2002. Total fruit and nut yields are fresh weights at harvest. Marketable nut yields are limited to split in-shell nuts dried to $5 \%$ moisture.

TABLE 2. The association between the level of infection by Verticillium dahliae and cumulative yield from pistachio trees planted on different rootstocks

\begin{tabular}{|c|c|c|c|c|c|c|}
\hline \multirow[b]{3}{*}{ Rootstock } & \multicolumn{6}{|c|}{ Extent of infection at termination of experiment ${ }^{\mathrm{x}}$} \\
\hline & \multicolumn{3}{|c|}{ Cumulative marketable yield ${ }^{y}$ (kg per female tree \pm SE) } & \multicolumn{3}{|c|}{ Cumulative total yield ${ }^{\mathrm{z}}(\mathrm{kg}$ per female tree $\pm \mathrm{SE})$} \\
\hline & Not infected & Mildly infected & Moderately or more infected & Not infected & Mildly infected & Moderately or more infected \\
\hline P. atlantica & $28 \pm 2$ & $29 \pm 2$ & $19 \pm 2$ & $100 \pm 9$ & $105 \pm 7$ & $69 \pm 6$ \\
\hline PGII hybrid & $22 \pm 3$ & $27 \pm 3$ & $16 \pm 1$ & $74 \pm 12$ & $91 \pm 12$ & $55 \pm 4$ \\
\hline UCBI hybrid & $28 \pm 1$ & $29 \pm 4$ & $26 \pm 3$ & $110 \pm 4$ & $113 \pm 14$ & $104 \pm 11$ \\
\hline
\end{tabular}

${ }^{\mathrm{x}}$ A cross-sectional disk of the trunk $5 \mathrm{~cm}$ above and below the graft union was polished, examined for symptoms of $V$. dahliae, and used for fungal isolation. Trees were rated as noninfected if the xylem was free of symptoms, moderately or highly infected if there were six or more lesions on either the rootstock or the scion, and mildly infected if the wood was symptomatic but did not meet the criterion for the moderate category. Only female trees that survived through 2002 are included. Analysis of variance and contrast analyses are shown in the text.

${ }^{\mathrm{y}}$ Marketable nut yields are limited to split in-shell nuts dried to 5\% moisture.

${ }^{z}$ Total yields are fresh weights at harvest. 
trees had the same visual ratings on both rootstock and scion: asymptomatic with no positive isolations; symptomatic with five or fewer lesions and a positive isolation; or symptomatic with six or more lesions and a positive isolation. Thirteen percent of the trees had all ratings in one of the three symptom-isolation categories, but the rating on the rootstock indicated more disease than the rating on the scion. Three percent of the trees had all ratings in one of the three symptom-isolation categories, but the ratings on the scion indicated more infection than that on the rootstock. Seven percent had symptomatic rootstocks and asymptomatic scions that both tested positive. One percent had symptomatic scions and asymptomatic rootstocks that both tested positive. No rootstock was disproportionately associated with one of these groups. There was only one tree with completely asymptomatic wood in which $V$. dahliae was isolated.

Because all female trees were grafted with the same clonal scion and the isolation data indicated that xylem symptoms provided an accurate diagnosis of infection (Tables 2 and 6), trees were rated as noninfected if the xylem was free of symptoms, moderately or more infected if there was six or more lesions on either the rootstock or the scion, and mildly infected if the wood was symptomatic but did not meet the criterion for the moderate category.

Rootstocks varied significantly $(P=0.0001)$ in trunk circumference, total fresh fruit and nut yield, and marketable yield (Table 1). UCBI and $P$. integerrima were similar and had significantly higher yield and trunk circumference than that of PGII. The surviving $P$. atlantica also performed significantly better than the surviving PGII.

In a two-factor ANOVA of the effect of rootstock and infection on cumulative marketable yield, there was a highly significant rootstock effect $(P=0.0001)$ (Table 2). Trees on PGII had a significantly $(P<0.05)$ lower marketable yield than trees on the other rootstocks. Level of infection also was highly significant $(P=$ $0.0001)$. Contrast analysis $(P=0.05)$ adjusted by a Bonferroni criterion $(P=0.016)$ indicated there were significant differences in cumulative marketable yield between moderately or more infected and the other two infection categories $(P=0.0008$ and 0.0001 for the contrast between moderately or more infected and either not infected or mildly infected, respectively). There were no differences in marketable yield between trees that were mildly infected versus noninfected $(P=0.14)$.

Although there was no significant $(P=0.25)$ rootstock-infection interaction in the cumulative marketable yield data discussed previously and shown in Table 2, the effect of infection on marketable yield was more pronounced in the two susceptible rootstocks than in the two resistant ones. In a separate one-way ANOVA for each rootstock, there were highly significant effects of infection on marketable yield for trees on P. atlantica and PGII $(P=0.002$ and 0.009 , respectively), but only a significant effect for trees on $P$. integerrima $(P=0.029)$ and no effect for those on UCBI $(P=0.8)$. A separate contrast analysis $(P=0.05)$ adjusted by a Bonferroni criterion $(P=0.025)$ for each rootstock in which there was a significant $F$ test indicated that there were no significant effects between not infected and mildly infected, but significant effects between not infected and moderately or more infected in only $P$. atlantica $(P=0.003)$. However, there were highly significant differences in cumulative marketable yield between mildly infected and moderately or more infected in trees on $P$. atlantica $(P=0.008)$, PGII $(P=0.005)$, and $P$. integerrima $(P=0.009)$ rootstocks.

A two-factor ANOVA of the effect of rootstock and infection on circumference (data not shown) and on cumulative total fruit and nut yield (Table 2) were generally similar to those shown for marketable yield. However, there were more significant $(P=$ $0.05)$ differences between rootstocks for cumulative total yield with trees on $\mathrm{UCBI} \equiv P$. integerrima $>P$. atlantica $>$ PGII. Trees that were rated as moderately or more infected had significantly less total yield than trees in other infection categories $(P=0.0009$ and 0.0001 for the contrast between moderately or more infected and either not infected or mildly infected, respectively). There were no differences in total yield of trees that were mildly infected compared with noninfected trees $(P=0.18)$. In a separate one-way ANOVA for each rootstock, there were significant effects of infection of trees on $P$. atlantica $(P=0.002)$, PGII $(P=$ $0.012)$, and $P$. integerrima $(P=0.03)$, but not UCBI. For each rootstock, results of contrast analysis of infection categories on total yield were very similar to those for marketable yield.

The correlation between results for marketable yield and total yield is supported by the fact that over the years rootstock did not

TABLE 3. The association between area and percentage of nondiscolored wood in a scion cross section and cumulative marketable yield of pistachio trees planted on different rootstocks in Verticillium dahliae-infested soil ${ }^{\mathrm{y}}$

\begin{tabular}{|c|c|c|c|c|c|c|}
\hline \multirow[b]{3}{*}{ Rootstock } & \multicolumn{6}{|c|}{ Linear regression on cumulative marketable yield ${ }^{\mathrm{z}}$} \\
\hline & \multicolumn{3}{|c|}{ Nondiscolored wood, $\mathrm{cm}^{2}$} & \multicolumn{3}{|c|}{ Nondiscolored wood, $\%$ of total } \\
\hline & Slope \pm SE & $R^{2}$ & $P$ value & Slope \pm SE & $R^{2}$ & $P$ value \\
\hline P. atlantica & $0.08 \pm 0.01$ & 0.49 & 0.0001 & $0.33 \pm 0.05$ & 0.48 & 0.0001 \\
\hline PGII & $0.07 \pm 0.01$ & 0.34 & 0.0001 & $0.26 \pm 0.07$ & 0.23 & 0.0003 \\
\hline UCBI & $0.04 \pm 0.02$ & 0.05 & 0.08 & $-0.03 \pm 0.1$ & 0.0008 & 0.8 \\
\hline
\end{tabular}

${ }^{y}$ A cross-sectional disk of the trunk $5 \mathrm{~cm}$ above the graft union was removed from 10-year-old trees. After the disks were polished, a digital image of the scion side was used to quantify the area and percentage of nondiscolored wood. Only female trees that survived through 2002 are included.

${ }^{\mathrm{z}}$ Marketable nut yields (in kilograms) are limited to split in-shell nuts dried to 5\% moisture.

TABLE 4. Cumulative marketable yield from female pistachio trees that survived through 2002 in a trial in Verticillium dahliae-infested soil in the San Joaquin Valley in California

\begin{tabular}{|c|c|c|c|c|c|c|c|}
\hline \multirow[b]{3}{*}{ Rootstock } & \multicolumn{4}{|c|}{ Tree vigor in 2002} & & & \\
\hline & Excellent & Good & Fair & Poor & \multicolumn{3}{|c|}{ Linear regression } \\
\hline & \multicolumn{4}{|c|}{ Marketable yield per tree, $\mathrm{kg} \pm \mathrm{SE}^{\mathrm{z}}$} & Slope \pm SE & $R^{2}$ & $P$ value \\
\hline$P$. atlantica & $23 \pm 3 \mathrm{ab}$ & $29 \pm 1 \mathrm{a}$ & $16 \pm 1$ & $9 \pm 2$ & $6.6 \pm 1.2$ & 0.38 & 0.0001 \\
\hline PGII & $28 \pm 4 \mathrm{ab}$ & $24 \pm 2 \mathrm{a}$ & $15 \pm 1$ & $9 \pm 1$ & $7.1 \pm 0.9$ & 0.53 & 0.0001 \\
\hline UCBI & $29 \pm 1 \mathrm{~b}$ & $28 \pm 1 \mathrm{a}$ & $22 \pm 6$ & 9 & $3.8 \pm 1.2$ & 0.14 & 0.003 \\
\hline
\end{tabular}

${ }^{z}$ Values within a column followed by the same letter are not significantly different according to Tukey's method. Text provides statistical details. - Indicates that there are no trees in the category. 
have an effect $(P>0.05)$ on percentage of any of the nut quality categories: undamaged split nuts, nonsplits, blanks, or damaged nuts (data not shown).

As an indirect estimate of relative fungal biomass in the scion, we quantified the area and percentage of discolored wood in each scion. Linear regressions of the area and percentage of nondiscolored wood on cumulative marketable yield are shown in Table 3. In $P$. atlantica and PGII, the slopes are highly significant $(P \leq$ $0.0003)$ with modest $R^{2}$ values $(0.23$ to 0.49$)$. In contrast, in $P$. integerrima and UCBI, the slopes are not significantly $(P \geq 0.08)$ different from zero.

Vigor was assessed visually by the relative appearance of the scions. Because P. integerrima had no trees in two categories of vigor, a two-factor ANOVA with only the other three rootstocks was performed. In a two-factor ANOVA on the effect of rootstock and vigor on marketable yield, when the effect of vigor was controlled, the rootstock effect was reduced to nonsignificance $(P=$ 0.066) (Table 4). When we controlled for the rootstock effect, tree vigor had a highly significant $(P=0.0001)$ effect on marketable yield. A second two-factor ANOVA with the four rootstocks and the two highest vigor ratings indicated a significant root-vigor interaction $(P=0.002)$. Trees that were rated good or excellent had indistinguishable $(P>0.05)$ marketable yields, except for $P$. integerrima in which trees rated as good had significantly greater marketable yields than those rated as excellent. There were no significant differences between rootstocks for the good category, but there were significant $(P=0.0005)$ differences between rootstocks in the excellent category. The association between vigor and yield was further examined by linear regression (Table 4). For a collective analysis, $P$. integerrima was excluded because of the lack of trees in two categories. A linear regression of marketable yield on the vigor of $P$. atlantica, PGII, and UCBI had an $R^{2}$ of 0.48 (slope $\left.=6.0 \pm 0.6 ; P=0.0001\right)$. There were no significant differences in slopes between the three rootstocks using a Bonferroni-adjusted $P$ value.

For trees on $P$. atlantica and PGII rootstocks, trees with either cankers or severely cracked trunks had significantly smaller yields than those without (Table 5).

The association between infection and vigor is shown in Table 6. Reducing the table into infected versus noninfected, 91 and $73 \%$ of the PGII and P. atlantica were infected, respectively. Sixty-five and twenty-five percent of the $P$. integerrima and UCBI were infected. Multiple comparisons based on a row effects log-linear model $(P=0.05$ criterion reduced to $P=0.0085)$ indicate that the percentage of infected trees on PGII and $P$. atlantica did not

TABLE 5. The association of trunk symptoms in 2002 with cumulative marketable yield from pistachio nut trees grown in a rootstock field trial in soil infested with Verticillium dahliae ${ }^{\mathrm{w}}$

\begin{tabular}{|c|c|c|c|c|}
\hline \multirow[b]{3}{*}{ Rootstock } & Trunk con & dition in $2002^{x}$ & \multirow{3}{*}{$\begin{array}{l}P \text { value of } \\
\text { trunk effect } \\
\text { for market- } \\
\text { able yield }\end{array}$} & \multirow{3}{*}{$\begin{array}{l}\text { Trees with } \\
\text { cankered or } \\
\text { severely } \\
\text { cracked } \\
\text { trunks, \% }\end{array}$} \\
\hline & Normal & $\begin{array}{c}\text { Cankered or } \\
\text { severely cracked }\end{array}$ & & \\
\hline & \multicolumn{2}{|c|}{$\begin{array}{l}\text { Marketable yield per tree, } \\
\qquad \mathrm{kg} \pm \mathrm{SE}\end{array}$} & & \\
\hline P. integerrima & $25 \pm 1 \mathrm{ab}$ & $25 \pm 1 \mathrm{~b}$ & $\mathrm{NS}^{\mathrm{z}}$ & $7 \mathrm{~b}$ \\
\hline$P$. atlantica & $27 \pm 1 \mathrm{~b}$ & $13 \pm 1 \mathrm{a}$ & 0.0001 & 35 a \\
\hline PGII hybrid & $21 \pm 2 \mathrm{a}$ & $14 \pm 1 \mathrm{a}$ & 0.0004 & $47 \mathrm{a}$ \\
\hline UCBI hybrid & $28 \pm 1$ & 9 & - & $2 \mathrm{~b}$ \\
\hline
\end{tabular}

w Only trees that survived through 2002 are included.

x A two-factor analysis of variance indicated a significant rootstock-trunk interaction. UCBI was not included in the comparison of least-squares means because there was only one tree with a symptomatic trunk. Values within a column followed by the same letter are not significantly different according to Tukey's method $(P=0.05)$.

${ }^{y}$ Data were analyzed by a row effects log-linear model. Values followed by the same letter are not significantly different $(P \geq 0.05)$ according to a Bonferroni-adjusted criterion $(P=0.0085)$.

${ }^{\mathrm{z}}$ Not significant, $P \geq 0.05$. differ, and $P$. atlantica and $P$. integerrima did not differ, but there was a significantly higher percentage of infected trees on PGII than on $P$. integerrima. UCBI had the lowest percent infected. Results were the same when infection was classified into three categories: not infected; mildly infected; and moderately or highly infected.

Results of the multiple comparisons of vigor between rootstocks were the same regardless of whether vigor was classified into two or four categories. All trees on $P$. integerrima rootstocks were rated as good or excellent, even though $48 \%$ of the trees were moderately or more infected. The log-linear models detected a significant difference between the vigor of trees on $P$. integerrima and those on UCBI; the difference first appeared in 1998, but did not appear to increase through 2002. The vigor of trees on $P$. atlantica was less than on either UCBI or $P$. integerrima. Trees on PGII had the least vigor.

Analysis of the data in Table 6 using a log-linear model supports the conclusion that, for each rootstock, the probability of having a noninfected tree with good or better vigor is greater than the probability of having a noninfected tree with fair or worse vigor. Similarly, the probability of having an infected tree with fair or worse vigor is greater than the probability of having a moderately or more infected tree with good or better vigor. Using trinomial categories (not infected, mildly infected, and moderately or highly infected) or binomial infection categories (not infected and infected), multiple comparisons of log-linear models indicated that $\mathrm{UCBI}>P$. integerrima $\geq P$. atlantica $>$ PGII $(P=$ 0.05 reduced using a Bonferroni-adjusted criterion). Using either quadrinomial vigor categories (excellent, good, fair, and poor) or binomial vigor categories (excellent or good and fair or poor), multiple comparisons of log-linear models indicated that $P$. integerrima $>\mathrm{UCBI} \geq P$. atlantica $>$ PGII $(P=0.05$ reduced using a Bonferroni-adjusted criterion).

Overall, the analysis indicates that for trees grown in $V$. dahliaeinfested soil, the observed data in Table 6 can be predicted reasonably accurately $(P>0.05)$ when a log-linear model incorporates the following associations: rootstock affects scion vigor; rootstock affects the extent to which the fungus is present in the trunk; and infection and scion vigor are inversely associated.

\section{DISCUSSION}

To our knowledge, this is the first report of a pistachio rootstock field trial through the first 5 years of nut production in soil

TABLE 6. The association between the level of infection by Verticillium dahliae as assessed by destructive sampling, and the vigor of the female pistachio nut trees grown on four different rootstocks in soil infested with V. dahliae

\begin{tabular}{llrcc}
\hline & & \multicolumn{3}{c}{ Level of infection $^{z}$} \\
\cline { 3 - 5 } & & $\begin{array}{c}\text { Not } \\
\text { infected }\end{array}$ & $\begin{array}{c}\text { Mildly } \\
\text { infected }\end{array}$ & $\begin{array}{c}\text { Moderately or } \\
\text { more infected }\end{array}$ \\
\cline { 3 - 5 } Rootstock & Vigor & Percentage of trees of each rootstock \\
\hline P. integerrima & Good or excellent & 35 & 17 & 48 \\
& Fair or poor & 0 & 0 & 0 \\
$P$. atlantica & Good or excellent & 27 & 12 & 23 \\
& Fair or poor & 0 & 0 & 38 \\
PGII hybrid & Good or excellent & 4 & 8 & 23 \\
& Fair or poor & 6 & 2 & 58 \\
UCBI hybrid & Good or excellent & 70 & 7 & 13 \\
& Fair or poor & 5 & 2 & 3 \\
\hline
\end{tabular}

y Both vigor and infection assessments were made in 2002.

$\mathrm{z}$ The independence of rootstock, vigor with the two levels shown, and infection (noninfected and infected) was tested using a log-linear model. The independence model was rejected $(P=0.0001)$; requisite terms for a model that could not be rejected $\left(P=0.18\right.$ by $\chi^{2} ; P=0.08$ by $\left.\mathrm{G}^{2}\right)$ indicates that rootstock significantly affects infection, rootstock affects vigor, and infection and vigor are negatively associated. 
naturally infested with $V$. dahliae. As expected, $P$. atlantica and $P$. integerrima performed as $V$. dahliae-sensitive and resistant rootstocks, respectively. Here we show that UCBI produced as much yield as $P$. integerrima. However, we note that whereas $100 \%$ of the trees on $P$. integerrima rootstocks were visually rated as either good or excellent in vigor, only $90 \%$ of the UCBI were similarly rated. In two other pistachio rootstock trials in different locations that have never shown symptoms of Verticillium wilt, UCBI either produced the highest or equivalent marketable yields with PGII, PGII produced greater than or equivalent yields to
$P$. integerrima, and $P$. atlantica produced the lowest yields (L. Ferguson, unpublished data). In addition to its resistance to Verticillium wilt, UCBI is frost-tolerant, in contrast to P. integerrima, which is frost-sensitive. UCBI also is more salt-tolerant than $P$. integerrima (10). Thus, we recommend UCBI as a pistachio rootstock for the San Joaquin Valley in California.

These data support the contention that most infections in $P$. vera "Kerman" scions have little effect on overall tree growth or productivity. Most of the pigmented material in the wood is presumably phenolics and/or tannins that are secreted by ray cells;
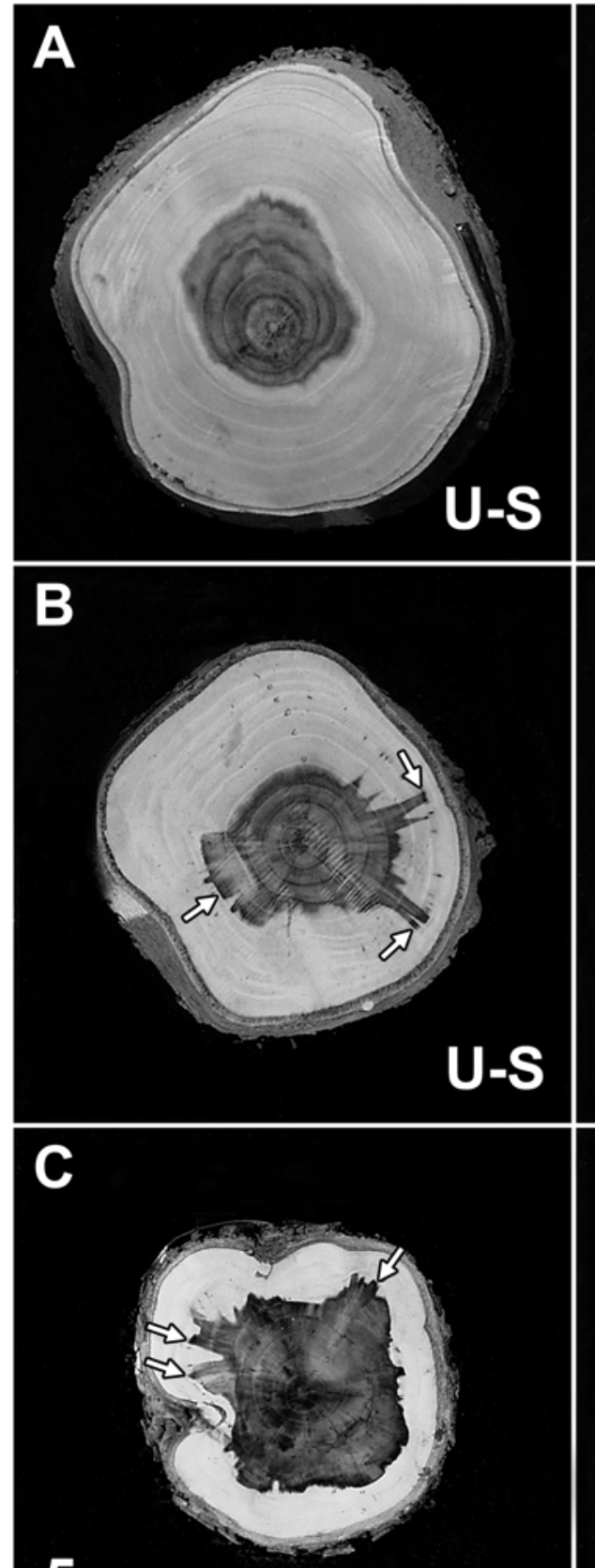

\section{$5 \mathrm{~cm}$}

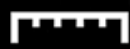

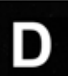

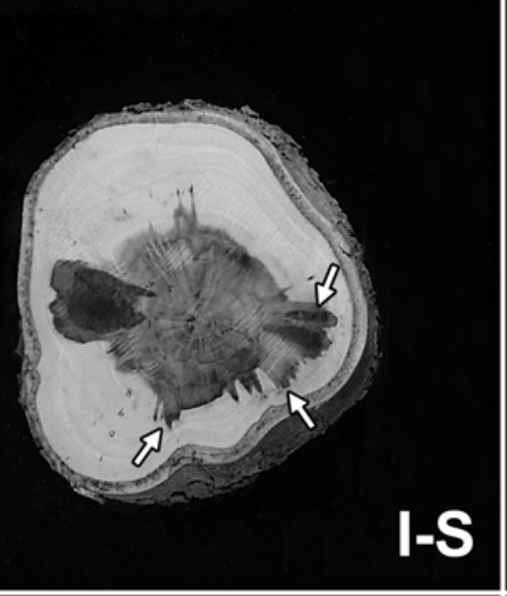

E

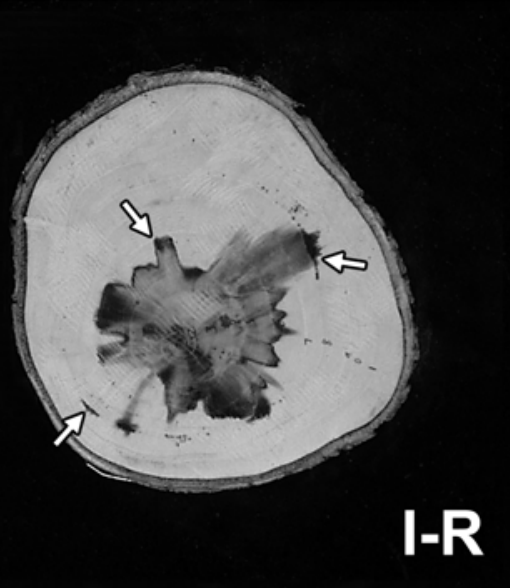

\section{$\mathbf{F}$}

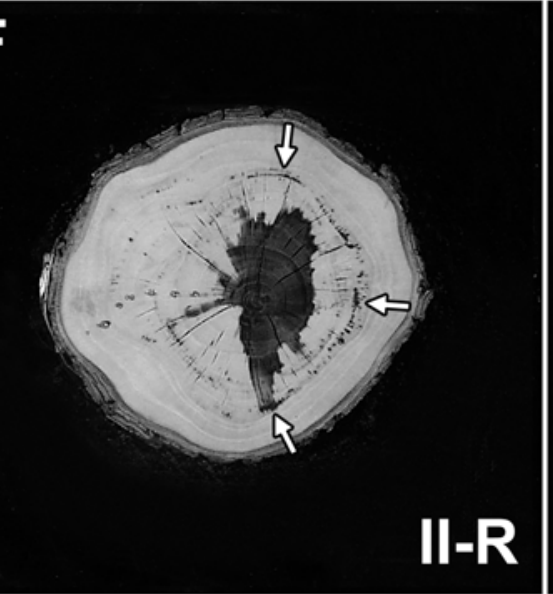

$\mathbf{G}$

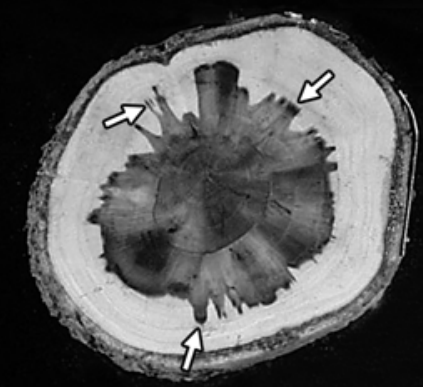

A-S

\section{H}

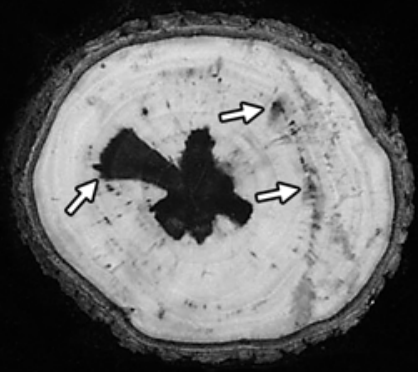

A-R

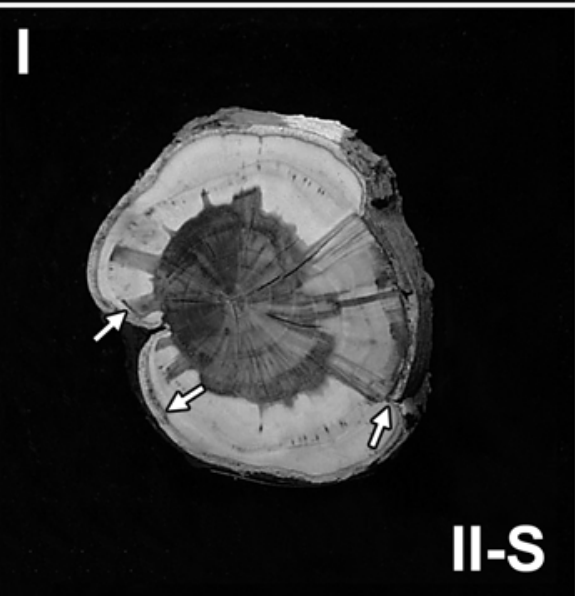

Fig. 1. Cross sections of trunks in the graft region of pistachio nut trees after a 10 -year rootstock trial in soil infested with Verticillium dahliae. Only female trees are shown here and all females were grafted with the same clone. Panel letters are shown in the upper left and the centimeter scale is the same for all panels. Letters in the lower right indicate the rootstock (U = 'UCBI'; I = Pistacia integerrima; A = P. atlantica; and II = 'PGII') followed by the side of the wood shown $(\mathrm{S}=$ scion; $\mathrm{R}=$ rootstock). Arrows indicate typical symptomatic regions from which $V$. dahliae was readily isolated. A, Scion of an uninfected tree on a (P. atlantica 'KAC' $\times$ P. integerrima) UCBI rootstock. B to I, Trees infected with $V$. dahliae and scored in the moderately or more infected category. B, Scion of a tree on a UCBI rootstock. C, Scion of a tree on a PGII rootstock. D to E, A tree on a $P$. integerrima rootstock. F, Rootstock of the tree in $\mathbf{C}$. $\mathbf{G}$ to $\mathbf{H}$, A tree on hybrid $P$. atlantica rootstock. I, Scion of a tree on a PGII rootstock in which $V$. dahliae caused a canker. 
the material may assist in host defense, but also can limit water transport if it is present in xylem that is conducting water. While the material is primarily a measure of the scion's response to infection, differences between genetically identical scions should be a function of the amount of fungal biomass in the scion tissue. Interestingly, while there was a highly significant association between the relative amount of nondiscolored wood and marketable yield for trees on the two susceptible cultivars, there was no association for trees on the two resistant rootstocks. Because the resistant $P$. integerrima and the susceptible $P$. atlantica rootstocks had similar percentages of infected scions (65 and 73\%, respectively), the production of discolored wood appears to be a resistance response that does not generally adversely affect growth or yield. Thus, the correlation between the marketable yield on both the area of nondiscolored wood and the percentage of nondiscolored wood for the two Verticillium wilt-sensitive rootstocks appears to be a consequence of a more fundamental difference between the Verticillium wilt-sensitive versus resistant rootstocks. These data suggest that $V$. dahliae negatively impacts growth and productivity of susceptible trees when it is in the root system, not when it enters the scions.

Overall, the results demonstrate that $V$. dahliae significantly reduced yield when trees on $P$. atlantica, PGII, and $P$. integerrima were "moderately or more" infected. However, the effect was more pronounced in $V$. dahliae-sensitive rootstocks than in V. dahliaeresistant rootstocks. Compared with the noninfected trees, the moderately or more infected trees on the $V$. dahliae-sensitive rootstocks $P$. atlantica and PGII had 32 and $27 \%$ lower yields. In comparison, moderately or more infected trees on the two $V$. dahliaeresistant rootstocks UCBI and $P$. integerrima had only 7 and $8 \%$ lower yields, respectively, compared with the noninfected controls. Indeed, there was no significant effect of infection on yield for UCBI, although there was for P. integerrima. Similarly, there was no significant association between either the area or the percentage of nondiscolored wood and marketable yield for trees on the two $V$. dahliae-resistant rootstocks. In contrast, there was a highly significant association between both the area and the percentage of nondiscolored wood and marketable yield for trees on the two $V$. dahliae-susceptible rootstocks. These data suggest that both UCBI and $P$. integerrima have some tolerance to $V$. dahliae in the sense that infected rootstocks maintain function.

In this trial, the reduced yield in moderately or more infected trees on $P$. atlantica and PGII rootstocks underestimated the economic impact of Verticillium wilt because it does not include the loss in yield from the dead trees. Although the course of the disease was variable and some trees appeared to die suddenly, other trees declined over years before death. The economic impact of tree death is exacerbated by the fact that a replacement pistachio tree requires 7 years to produce a reasonable yield.

There was no significant difference in yields between noninfected trees and "mildly" infected trees. However, yields of mildly infected trees were always higher, although not significantly, than that of noninfected trees (4 to 23\%), depending on the rootstock. Thus, the lack of a significant yield effect with mildly infected trees may be due to a stimulation of nut production; Verticillium wilt in almonds and landscape trees is often accompanied by increased nut production $(2,5,9)$. It is also possible that 10 -year-old trees that are mildly infected may show declines in yield over time. Pistachios are long lived, i.e., 700-year-old productive trees have been reported (25).

Verticillium wilt in pistachios is most clearly diagnosed by an acute "strike" symptom. A chronic condition called "thin leaf decline" in pistachios is also diagnostic. In other tree species $(2,24)$, Verticillium wilt is associated with a nonspecific reduction in vigor. Our data suggest that infection is associated with a nonspecific reduction in vigor in the two $V$. dahliae-sensitive rootstocks. Observations of the cross sections of wood (Fig. 1I) along with fungal isolation indicate that $V$. dahliae also can cause cankers and severe cracking in pistachio tree trunks. We suggest that if $V$. dahliae is present in the most recent annual ring, the cambium, phloem, or both may be killed by the fungus, or perhaps more likely, the pigmented material secreted by the xylem ray cells in response to the fungus.

Although we only isolated $V$. dahliae from one entirely asymptomatic piece of wood, we isolated $V$. dahliae from some asymptomatic portions of wood. Thus, $V$. dahliae does not appear to be entirely limited to the symptomatic regions. On the scions in which the ray cells released pigmented material, the pattern of pigmentation is consistent with the hypothesis that $V$. dahliae grows into the older wood, but not into the newer wood. In olive and apricot trees, at least in some locations, $V$. dahliae is difficult to isolate from the trunks in hot weather $(14,27)$. This finding led to the conclusion that infection in the trunk was maintained by annual reinfection. Although we did all of our isolations during a single time period, the frequency with which we isolated the fungus from symptomatic regions suggests that infections in the trunk are perennial in pistachios in this location.

$P$. integerrima is the current $V$. dahliae-"resistant" standard in the field. Indeed, in this 10-year trial in $V$. dahliae-infested soil, all of the trees on $P$. integerrima rootstocks appeared to be in either excellent or good health. This is despite the fact that during the 10 -year trial, $11 \%$ of the trees on P. integerrima rootstock had had a branch with a symptomatic strike, but generally in only 1 year. Destructive sampling of the trees in 2002 showed that $65 \%$ of the trees on $P$. integerrima rootstocks had infections in the scions, which statistically was not less than in the V. dahliae-sensitive standard $P$. atlantica. Thus, $P$. integerrima is tolerant to $V$. dahliae, in the sense that even though there is a high probability of penetration of the fungus into the scion, the scion is generally asymptomatic and infected rootstocks maintain excellent function. For trees on P. integerrima rootstocks, even the $7 \%$ with cankers on their trunks maintained productivity. Many crops have cultivars that tolerate $V$. dahliae, including cotton, eggplant, tomato, potato, and strawberries $(7,13,18)$. In contrast, a diversity of other plant species appears to resist infections by $V$. dahliae by either restricting fungal access into or translocation within the xylem $(6,8)$. In pistachios, significantly fewer trees on the UCBI rootstocks were infected around the graft union than in P. integerrima $(25 \%$ versus $65 \%)$. In addition, there was a significant difference in the visual appearance of trees on $P$. integerrima versus UCBI rootstocks; in 2002, all of the trees on P. integerrima were visually rated as good or excellent, whereas $10 \%$ of the trees on UCBI were rated as fair or poor. Throughout the 10-year trial, there was no evidence of any pest, abiotic condition, or pathogen other than $V$. dahliae that was affecting the trees. These data are consistent with the hypothesis that UCBI and P. integerrima have at least one genetic difference in how they interact with $V$. dahliae; and that $P$. integerrima primarily tolerates $V$. dahliae, whereas UCBI avoids colonization of its xylem by $V$. dahliae to a greater extent than $P$. integerrima.

Although UCBI and PGII are both interspecific hybrids of a $P$. atlantica female and a $P$. integerrima male, the difference in response to $V$. dahliae may be due to genetic diversity in the parents of each rootstock. Morgan et al. (20) detected a resistance-type response to $V$. dahliae in $P$. atlantica 'KAC' shoots from the tree used as the female parent for production of UCBI seed, but a susceptible response in shoots from the more typical $P$. atlantica used in commercial production. In addition, leaf shape differs between $P$. atlantica 'KAC' and the $P$. atlantica used to produce commercial seed.

The hybrid PGII may be more susceptible to V. dahliae in comparison to its parental types, $P$. atlantica and $P$. integerrima. As noted previously, in two trials in which Verticillium wilt was not observed in any of the trees, the growth and productivity of PGII was better than or equivalent to $P$. integerrima and better than $P$. atlantica (L. Ferguson, unpublished data). In the current trial, 
trees on PGII had significantly lower marketable yields than trees on any of the other rootstocks, even though the trees on PGII rootstocks that were rated as excellent yielded as well as any of the rootstocks. In the 1990 field trial, PGII had significantly higher disease ratings and a higher percentage of symptomatic plants than $P$. atlantica (20). Greater susceptibility in hybrids than in either parent has been reported in other pathosystems including walnut (17) and willow trees $(11,26)$.

Our ANOVA and log-linear model analyses indicate that in soil infested with $V$. dahliae, three associations significantly affect pistachio nut yield. Rootstock affects both scion vigor and the extent to which the fungus is present in the trunk. In addition, infection and scion vigor are inversely associated.

\section{ACKNOWLEDGMENTS}

This research was partly supported by grants from the California Pistachio Commission. UCBI seedlings were originally selected by L. J. Ashworth, Jr. and D. P. Morgan. We thank S. Bassein for statistical advice; B. Hanson and A. Alzondo of Paramount Processing for assessing nut quality; D. Spencer for access to equipment for image analysis; H. Reyes, G. Dhahan, H. Guo, and J. Liebman for field and laboratory assistance; and J. Ross and his staff at the West Side Field Station.

\section{LITERATURE CITED}

1. Agresti, A. 1990. Categorical Data Analysis. John Wiley \& Sons, New York.

2. Ash, C. L. 2001. Verticillium wilt of trees: A brief history and current research. Pages 149-156 in: Shade Tree Wilt Diseases. C. L. Ash, ed. The American Phytopathological Society, St. Paul, MN.

3. Ashworth, L. J., Jr., and Gaona, S. A. 1982. Evaluation of clear polyethylene mulch for controlling Verticillium wilt in established pistachio nut groves. Phytopathology 72:243-246. (Errata Figure 1 published in Phytopathology 73:1142).

4. Ashworth, L. J., Jr., and Zimmerman, G. 1976. Verticillium wilt of the pistachio nut tree: Occurrence in California and control by soil fumigation. Phytopathology 66:1449-1451.

5. Blanchard, R. O., and Tatter, T. A. 1981. Field and Laboratory Guide to Tree Pathology. Academic Press, New York.

6. Cooper, R. M. 2000. Verticillium-host interactions: Past achievements and future molecular prospects. Pages 144-150 in: Advances in Verticillium Research and Disease Management. E. C. Tjamos, R. C. Rowe, J. B. Heale, and D. R. Fravel, eds. The American Phytopathological Society, St. Paul, MN.

7. Dan, H., Ali-Khan, S. T., and Robb, J. 2001. Use of quantitative PCR diagnostics to identify tolerance and resistance to Verticillium dahliae in potato. Plant Dis. 85:700-705.

8. Davis, J. R., Huisman, O. C., Sorensen, L. H., and Schneider, A. T. 2000. Field studies comparing the susceptibility of various crops to colonization by Verticillium dahliae. Pages 311-314 in: Advances in Verticillium Research and Disease Management. E. C. Tjamos, R. C.
Rowe, J. B. Heale, and D. R. Fravel, eds. The American Phytopathological Society, St. Paul, MN.

9. Epstein, L., MacDonald, J., and Stapleton, J. J. 2002. Verticillium wilt in pistachios, almonds, walnuts, pecans and chestnuts. Pages 7-8 in: Compendium of Nut Crop Diseases in Temperate Zones. B. Teviotdale, T. J. Michailides, and J. W. Pscheidt, eds. The American Phytopathological Society, St. Paul, MN.

10. Ferguson, L., Poss, J. A., Grattan, S. R., Grieve, C. M., Wang, D., Wilson, C., Donovan, T. J., and Chao, C.-T. 2002. Pistachio rootstocks influence scion growth and ion relations under salinity and boron stress. J. Am. Soc. Hortic. Sci. 127:194-199.

11. Fritz, R. S., Nichols-Orians, C. M., and Brunsfeld, S. J. 1994. Interspecific hybridization of plants and resistance to herbivores: Hypotheses, genetics, and variable responses in a diverse herbivore community. Oecologia 97:106-117.

12. Gabriel, K. R. 1966. Simultaneous test procedures for multiple comparisons on categorical data. J. Am. Statist. Assoc. 61:1081-1096.

13. Garas, N. A., Wilhem, S., and Sagen, J. E. 1986. Relationship of cultivar resistance to distribution of Verticillium dahliae in inoculated cotton plants and to growth of single conidia on excised stem segments. Phytopathology 76:1005-1010.

14. Harrison, A. F., and Clare, B. G. 1970. Host reactions involved in the recovery of apricot trees from Verticillium rot. Aust. J. Biol. Sci. 23:1027-1032.

15. Huisman, O. C., and Ashworth, L. J., Jr. 1974. Quantitative assessment of Verticillium albo-atrum in field soils: Procedural and substrate improvements. Phytopathology 64:1043-1044.

16. Mandansky, A. 1988. Prescriptions for Working Statisticians. SpringerVerlag, New York.

17. McKenna, J. R., and Epstein, L. 2003. Relative susceptibility of Juglans species and interspecific hybrids to Agrobacterium tumefaciens. HortScience 38:435-439.

18. Melero-Vara, J. M., Blanco-Lopez, M. A., Bejarano-Alcazar, J., and Jimenez-Diaz, R. M. 1995. Control of Verticillium wilt of cotton by means of soil solarization and tolerant cultivars in southern Spain. Plant Pathol. 44:250-260.

19. Miller, R. G., Jr. 1981. Simultaneous Statistical Inference. 2nd ed. Springer-Verlag, New York.

20. Morgan, D. P., Epstein, L., and Ferguson, L. 1992. Verticillium wilt resistance in pistachio rootstock cultivars: Assays and an assessment of two interspecific hybrids. Plant Dis. 76:310-313.

21. Ogawa, J. M., and English, H. 1991. Diseases of Temperate Zone Tree Fruit and Nut Crops. Univ. Calif. Div. Agric. Nat. Res. Publ. 3345.

22. Pegg, G. F., and Brady, B. L. 2002. Verticillium Wilts. CAB International, Oxon, UK.

23. Raabe, R. D., and Wilhelm, S. 1978. Susceptibility of several Pistacia spp. to Verticillium albo-atrum. Plant Dis. Rep. 62:672-673.

24. Sánchez Hernández, M. E., Ruiz Dávila, A., Pérez de Algaba, A., Blanco López, M. A., and Trapero Casas, A. 1998. Occurrence and etiology of death of young olive trees in southern Spain. Eur. J. Plant Pathol. 104:347-357.

25. Whitehouse, W. E. 1957. A new crop for the western United States. Econ. Bot. 11:281-321.

26. Whitham, T. G. 1989. Plant hybrid zones as sinks for pests. Science 244:1490-1493.

27. Wilhelm, S., and Taylor, J. B. 1965. Control of Verticillium wilt of olive through natural recovery and resistance. Phytopathology 55:310-316. 